\title{
Fatores de risco que levam o câncer do colo do útero: Uma revisão integrativa
}

\section{Risk factors that lead to cervical cancer: An integrative review}

Factores de riesgo que llevan al cáncer de cuello uterino: Una revisión integradora

Sabrina Sousa Barros

ORCID: https://orcid.org/0000-0003-4517-0401

Cristo Faculdade do Piauí, Brasil

E-mail: sabrinabarros1901@gmail.com

Amanda Kelly Ferreira Resende ORCID: https://orcid.org/0000-0003-3855-4028 Cristo Faculdade do Piauí, Brasil E-mail: amandahkelly2015@gmail.com Dariely de Oliveira Silva ORCID: https://orcid.org/0000-0002-9281-7311 Cristo Faculdade do Piauí, Brasil

E-mail: darielyoliveira2016@gmail.com Marcelo da Silva ORCID: https://orcid.org/0000-0003-4509-8294 Cristo Faculdade do Piauí, Brasil

E-mail: marceloenf21@gmail.com

Marcos Roberto Nascimento Sousa ORCID: https://orcid.org/0000-0003-1634-5276 Cristo Faculdade do Piauí, Brasil

E-mail: marcosrobertoenfpi@gmail.com Ana Paula Melo Oliveira

ORCID: https://orcid.org/0000-0003-3328-9624 Cristo Faculdade do Piauí, Brasil E-mail: apmeloenfer@gmail.com

Sara Samara Ferreira de Araújo ORCID: https://orcid.org/0000-0003-2638-1094 Cristo Faculdade do Piauí, Brasil E-mail: sarasam231@gmail.com Anderson Pereira Freitas ORCID: https://orcid.org/0000-0003-2899-6148 Cristo Faculdade do Piauí, Brasil

E-mail: andersonft10tb@gmail.com

Aloíso Sampaio Souza

ORCID: https://orcid.org/0000-0002-5924-7994

Universidade Federal do Maranhão, Brasil E-mail: aloisofilho@hotmail.com

Guilherme Martins Gomes Fontoura ORCID: https://orcid.org/0000-0001-5430-0728

Universidade Federal do Maranhão, Brasil E-mail: Guilherme.fontoura@ discente.ufma.br

Evanielle Souza Andrade

ORCID: https://orcid.org/0000-0002-3089-9593

Ensino Superior do Vale do Parnaíba, Brasil

E-mail: evaniellesouzaandrade @ gmail.com

Danilo Sampaio Souza

ORCID: https://orcid.org/0000-0002-0216-3669

Centro Universitário Uninovafapi, Brasil

E-mail: danilosampaio16@hotmail.com

Flávia Maria Martins Melo

ORCID: https://orcid.org/0000-0001-7193-7333

Faculdade Luciano Feijão, Brasil

E-mail: flaviammelo@hotmail.com

Gabriel Mauriz de Moura Rocha

ORCID: https://orcid.org/0000-0002-1515-6951

Universidade Camilo Castelo Branco, Brasil E-mail: mauriz45@hotmail.com

Evaldo Sales Leal

ORCID: https://orcid.org/0000-0002-1424-9048

Universidade Federal do Piauí, Brasil

E-mail: evaldosleal@hotmail.com 


\begin{abstract}
Resumo
O câncer do colo do útero, conhecido também como cervical, é considerado um importante problema de saúde que atinge as mulheres em todos os continentes. Diante disso, surgiu a seguinte problemática: quais fatores de risco que contribuem para o desenvolvimento do câncer do colo do útero? Objetiva-se avaliar os fatores de risco que levam ao câncer do colo do útero. A presente pesquisa trata-se de uma revisão integrativa, em que foi realizada mediante as seguintes bases de dados: Biblioteca Virtual em saúde (BVS), LILACS, SCIELO, MEDLINE e BDENF. As palavras chave utilizadas seguiram a descrição dos termos Descritores em Ciências da Saúde (DECS). Foram elas: Fatores, riscos, neoplasias do colo do útero. Para a seleção dos estudos, os critérios de inclusão utilizados foram artigos, de (2014-2019), disponibilizados de forma integral e com livre acesso ao texto e que estivessem adequados ao tema proposto e estudos na língua portuguesa. De acordo com os achados foi possível constatar que os principais fatores envolvidos no desencadeamento do câncer de colo de útero foram: ser tabagistas ou ex-tabagistas, baixa renda, percepção negativa da própria saúde, uso do álcool, baixa escolaridade e renda familiar mensal, precocidade da coitarca, não uso do preservativo, paridade, baixa cobertura do rastreamento, ser solteira e falta de acesso ao serviço de saúde. Os objetivos do estudo foram alcançados de forma satisfatória, à medida que os resultados obtidos mostraram integralmente os fatores envolvidos com o câncer uterino. Um dos desafios encontrados foi a escassez de artigos relacionado ao tema.
\end{abstract}

Palavras-chave: Fatores; Riscos; Neoplasias do colo do útero.

\begin{abstract}
Cervical cancer, also known as cervical cancer, is considered a major health problem that strikes as women on every continent. Given this, the following problem arose: which risk factors contribute to the development of cervical cancer? The objective is to evaluate the risk factors that lead to cervical cancer. This research deals with an integrative review, which was performed using the following databases: Virtual Health Library (VHL), LILACS, SCIELO, MEDLINE and BDENF. The keywords used follow a description of the terms Health Sciences Descriptors (DECS). They were: Factors, Risks, Cervical Neoplasms. For the selection of studies, candidates for inclusion of selected articles (2014-2019), made available in full and with free access to the text and who are familiar with the topic and studies of Portuguese language. According to the findings, it was found that the main factors involved in the onset of cervical cancer were: smokers or former smokers, low income, negative perception of their own health, alcohol use, low education and monthly family income, precocity, not using condoms, parity, low screening coverage, being single and without access to health services. The objectives of the study were satisfactorily achieved, as the results were fully detected by the factors involved in uterine cancer. One of the challenges encountered was the scarcity of related articles.
\end{abstract}

Keywords: Factors; Risks; Uterine cervical neoplasms.

\title{
Resumen
}

El cáncer de cuello uterino, también conocido como cáncer de cuello uterino, se considera un problema de salud importante que afecta a las mujeres en todos los continentes. Ante esto, surgió el siguiente problema: ¿qué factores de riesgo contribuyen al desarrollo del cáncer de cuello uterino? El objetivo es evaluar los factores de riesgo que conducen al cáncer de cuello uterino. Esta investigación es una revisión integradora, en la que se realizó utilizando las siguientes bases de datos: Biblioteca Virtual en Salud (BVS), LILACS, SCIELO, MEDLINE y BDENF. Las palabras clave utilizadas siguieron la descripción de los términos Descriptores de Ciencias de la Salud (DECS). Fueron: Factores, riesgos, neoplasias del cuello uterino. Para la selección de los estudios, los criterios de inclusión utilizados fueron artículos, de (2014-2019), disponibles en su totalidad y con libre acceso al texto y que fueran adecuados a la temática y estudios propuestos en portugués. De acuerdo con los hallazgos, se encontró que los principales factores involucrados en la aparición del cáncer de cuello uterino fueron: ser fumadora o exfumadora, bajos ingresos, percepción negativa de la propia salud, consumo de alcohol, baja escolaridad e ingresos familiares mensuales, precocidad de coitarca, no uso de condón, paridad, baja cobertura de tamizaje, ser soltero y falta de acceso a servicios de salud. Los objetivos del estudio se alcanzaron satisfactoriamente, ya que los resultados obtenidos mostraron plenamente los factores implicados en el cáncer de útero. Uno de los desafíos encontrados fue la escasez de artículos relacionados con el tema.

Palabras clave: Factores; Riesgo; Neoplasias del cuello uterino.

\section{Introdução}

O câncer do colo do útero, conhecido também como cervical, é considerado um importante problema de saúde que atinge as mulheres em todos os continentes. É causado pela infecção de alguns tipos do Papiloma vírus Humano - HPV, especialmente os subtipos 16 e 18. A infecção genital por este vírus é muito frequente e não causa doença na maioria das vezes. No entanto, em determinados casos podem manifestar-se em alterações celulares que poderão evoluir para o câncer. Seu desenvolvimento, na maioria dos casos, se dá de forma lenta, podendo ser susceptível de prevenção e controle por meio da 
triagem e do tratamento precoce (Carmo Rocha, Bustamante Teixeira, Azevedo e Silva, de Castro Dias e Salim Miranda Duque, 2014).

O rastreamento da população feminina utilizando do exame de Papanicolau, também chamado de exame de prevenção do câncer do colo do útero (PCCU), pode reduzir em $80 \%$ o índice de mortalidade por câncer. Pesquisas executadas sobre mortalidade entre as mulheres demonstram que, apesar de haver uma queda nas taxas, sua incidência continua elevada, cerca de duas vezes maior em países menos desenvolvidos. A taxa de mortalidade é alta nas mais variadas faixas etárias, tendo como maior pico de incidência, o carcinoma in situ que acomete principalmente mulheres entre 25 e 40 anos e o carcinoma invasor, com prevalência na faixa etária de 48 a 55 anos (Brasil, 2017).

Uns dos principais motivos para a não realização do exame preventivo estar no desconhecimento por grande parte das mulheres, em que, na maioria dos casos procuram realizar o exame apenas quando há sinais e sintomas. Mostrando e reafirmando o desconhecimento das mesmas sobre a importância do exame em questão (Vukovic, Antic, Vasiljevic, Antic \& Matejic, 2015).

O PCCU deve ser realizado por toda mulher que tem ou já teve atividade sexual e estiver na faixa etária de 25 aos 64 anos de idade. Inicialmente, um exame deve ser feito a cada ano e, caso dois exames seguidos apresentem resultado normal, o exame pode passar a ser feito a cada três anos.

No caso das mulheres que nunca realizaram o Papanicolau e com mais de 64 anos, devem ser feitos dois exames preventivos no intermédio de um a três anos. Se os dois resultados forem negativos, essas mulheres poderão ser dispensadas de exames adicionais. Na maioria das mulheres a infecção pelo HPV não apresenta sintomas. Em determinados casos, o HPV pode ficar latente de meses a anos, sem manifestar sinais visíveis, ou apresentar manifestações subclínicas, ou seja, não visíveis a olho nu. Nos casos em que a diminuição da resistência do organismo pode desencadear a multiplicação do HPV e, com isso, provocar o aparecimento de lesões.

A medida mais eficaz para prevenção contra a infeção, é a vacina distribuída gratuitamente pelo SUS e está disponível para a população do sexo feminino e masculino com idade de 9 a 14 anos e 11 a 14 anos respectivamente. A vacinação é indicada também para as pessoas vivendo com HIV e transplantadas na faixa etária de 9 a 26 anos, fazendo-se necessário enfatizar sobre o uso de preservativos, sendo uma importante forma de prevenção do HPV, presente em $90 \%$ dos casos de câncer uterino e tem importante papel no desenvolvimento da neoplasia do colo e suas lesões precursoras (Brasil, 2015). Diante disso, surgiu a seguinte problemática: quais fatores de risco que contribuem para o desenvolvimento do câncer do colo do útero?

A partir disso podemos destacar a atuação da estratégia saúde da família no âmbito saúde da mulher, visto a maior proximidade com as mesmas. Dessa forma, os profissionais podem atuar como ferramenta para o esclarecimento e compreensão em relação à importância da realização do Papanicolau. Diante disso, o trabalho tem como objetivo avaliar os fatores de risco que levam ao câncer do colo do útero.

\section{Metodologia}

A revisão integrativa é um método que tem como finalidade sintetizar resultados obtidos em pesquisas sobre um tema ou questão, de maneira sistemática, ordenada e abrangente. É denominada integrativa porque fornece informações mais amplas sobre um assunto/problema, constituindo, assim, um corpo de conhecimento. Deste modo, o revisor/pesquisador pode elaborar uma revisão integrativa com diferentes finalidades, podendo ser direcionada para a definição de conceitos, revisão de teorias ou análise metodológica dos estudos incluídos de um tópico particular (Ercole, Melo, Alcoforado, 2014).

Segundo Soares, Hoga, Peduzzi, Sangaleti, Yonekura e Silva (2014), uma revisão integrativa pode ser desenvolvida mediante o seguimento de seis etapas, as quais são: identificação do tema e seleção da questão da pesquisa, coleta de dados e 
estabelecimentos de critérios de inclusão e exclusão, identificação de estudos pré-selecionados e selecionados, caracterização de estudos selecionados, análise e interpretação de resultados, apresentação e síntese do conhecimento.

A busca da presente pesquisa foi realizada mediante as seguintes bases de dados: Biblioteca Virtual em saúde (BVS), LILACS, SCIELO, MEDLINE e BDENF. As palavras chave utilizadas seguiram a descrição dos termos Descritores em Ciências da Saúde (DECS). Foram elas: Fatores, riscos, neoplasias do colo do útero.

Com base nisso, para a seleção dos estudos, os critérios de inclusão utilizados foram artigos, de (2014-2019), com a proposta de pesquisa envolvida em estudos disponibilizados de forma integral e com livre acesso ao texto e que estivessem adequados ao tema proposto e estudos na língua portuguesa. Para a exclusão dos artigos os critérios aplicados foram estudos que não estivesse de acordo com o tema proposto, artigos de revisão, artigos que estavam fora do período determinado pelos critérios de inclusão e no caso de duplicidade dos estudos nas devidas bases de dados, preservou-se apenas o estudo de uma base.

\section{Resultados e Discussão}

Inicialmente foi construído um quadro para expor os estudos contendo informações como título, autores e ano de publicação da pesquisa. Após as pesquisas nas bases de dados por meio dos descritores e após a aplicação dos critérios de inclusão e exclusão estabelecidos, foram selecionados 5 artigos presentes no Quadro 1 para análise da revisão integrativa.

Quadro 1. Artigos selecionados para análise de revisão integrativa.

\begin{tabular}{|c|c|c|c|}
\hline ORDEM & AUTORES & ANO & LOCAL \\
\hline $\mathbf{1}$ & Rozario, Silva, Koifman e Silva & 2019 & Juiz de Fora- Minas Gerais \\
\hline $\mathbf{2}$ & $\begin{array}{c}\text { Lombelo-Campos, Neves, Duque, Guerra e } \\
\text { Teixeira }\end{array}$ & 2018 & Ceará \\
\hline $\mathbf{3}$ & Nicolau, Aquino, Ximenes e Pinheiro & 2015 & Belo Horizonte \\
\hline $\mathbf{4}$ & Macêdo, Silva, Soares, Rosal, Carvalho e \\
Rocha, & 2015 & Teresina-PI \\
\hline
\end{tabular}

Fonte: Autores (2019).

Considerando os cinco artigos no quadro acima escolhidos e que compõe a amostra, observou-se que todas as publicações ocorreram no período de 2014 a 2019, todos são trabalhos completos, disponíveis de forma integral e grátis e na língua portuguesa. A escolha dos estudos demonstrados no quadro deu-se a qualidade e a proposta que se encaixa no tema proposto, pois trata-se de uma área específica da enfermagem que abrange artigos aos quais mantiveram a qualidade do trabalho e que procuraram identificar quais os benefícios as terapias manuais poderiam trazer aos praticantes de futebol em geral, realizando a comparação entre os mesmos. No Quadro 2 é possível observar o objetivo principal de cada artigo selecionado para a análise. 
Quadro 2. Objetivos dos artigos selecionados para análise de revisão integrativa.

\begin{tabular}{|c|c|c|}
\hline ORDEM & TÍTULO & OBJETIVOS \\
\hline 1 & $\begin{array}{l}\text { Caracterização de mulheres com câncer cervical } \\
\text { atendidas no Inca por tipo histológico }\end{array}$ & $\begin{array}{l}\text { Determinar a distribuição das características sociodemográficas, } \\
\text { reprodutivas, clínicas e de hábitos de vida na coorte de mulheres } \\
\text { diagnosticadas com câncer cervical, atendidas no Inca entre } 2012 \\
\text { e 2014, segundo o tipo histológico. }\end{array}$ \\
\hline 2 & $\begin{array}{l}\text { Fatores associados ao risco de alterações no } \\
\text { exame citopatológico do colo do útero }\end{array}$ & $\begin{array}{l}\text { Estimar a prevalência e avaliar os fatores associados ao alto risco } \\
\text { de apresentar alterações no exame citopatológico do colo do } \\
\text { útero em mulheres cobertas pela Estratégia de Saúde da Família. }\end{array}$ \\
\hline 3 & $\begin{array}{l}\text { Determinantes sociais proximais relacionados ao } \\
\text { câncer cervicouterino em mulheres privadas de } \\
\text { liberdade }\end{array}$ & $\begin{array}{l}\text { Analisar os determinantes sociais de saúde proximais, } \\
\text { considerados fatores de risco para a neoplasia cervicouterina, de } \\
\text { mulheres privadas de liberdade segundo o nível } 2 \text { do Modelo de } \\
\text { Atenção às Condições Crônicas (MACC). }\end{array}$ \\
\hline 4 & Infecção pelo HPV na adolescente & $\begin{array}{l}\text { Levantar dados e informações sobre as consequências da } \\
\text { infecção por HPV na adolescência, seu diagnóstico e condutas a } \\
\text { serem tomadas. }\end{array}$ \\
\hline 5 & $\begin{array}{l}\text { Fatores de risco e proteção à saúde de mulheres } \\
\text { para prevenção do câncer uterino }\end{array}$ & $\begin{array}{l}\text { Investigar os fatores de risco e de proteção de mulheres que } \\
\text { acessam o serviço de saúde para realização do exame preventivo } \\
\text { de câncer de colo uterino. }\end{array}$ \\
\hline
\end{tabular}

Fonte: Autores (2019).

Quadro 3. Demonstrativo dos principais achados dos estudos analisados na revisão integrativa.

\begin{tabular}{|c|l|}
\hline Ordem & \multicolumn{1}{c}{ Principais Achados } \\
\hline $\mathbf{1}$ & $\begin{array}{l}\text { Houve uma predominância de mulheres não brancas (67,4\%), com menos de } 8 \text { anos de estudo (51,9\%), com início } \\
\text { da atividade sexual até } 16 \text { anos de idade }(40,7 \%) \text {, histórico de gestação }(95,5 \%), \text { com mais de uma gestação (82,9\%) } \\
\text { e mais de dois filhos }(52,7 \%) \text {. Neste estudo, 45,8\% das mulheres com câncer de colo uterino eram tabagistas ou ex- } \\
\text { tabagistas }\end{array}$ \\
\hline $\mathbf{2}$ & $\begin{array}{l}\text { Constatou-se que as mulheres com baixa renda (RP = 1,12; IC95\%: 1,04-1,21), com percepção negativa da própria } \\
\text { saúde (RP = 1,13; IC95\%: 1,06-1,20), que faziam uso de tabaco (RP = 1,14; IC95\%: 1,05-1,23) ou álcool (RP = } \\
1,09 ; \text { IC95\%: 1,02-1,18) apresentaram alto risco de apresentar um exame alterado, ao passo que a idade elevada (RP } \\
=0,83 ; \text { IC95\%: 0,73-0,95) associou-se à diminuição desse risco. }\end{array}$ \\
\hline $\mathbf{3}$ & $\begin{array}{l}\text { Os principais fatores encontrados direcionaram-se para a baixa escolaridade e renda familiar mensal, precocidade da } \\
\text { coitarca, não uso do preservativo, paridade, baixa cobertura do rastreamento e hábitos tabagista }\end{array}$ \\
\hline $\mathbf{4}$ & $\begin{array}{l}\text { Embora o vírus HPV possa acometer pessoas de qualquer idade, é mais frequente em mulheres jovens, no período } \\
\text { de maior atividade sexual. Na adolescência, as taxas de prevalência cumulativa da infecção são altas, chegando a } \\
\text { atingir 82\% das adolescentes em populaçães seletas. }\end{array}$ \\
\hline $\mathbf{5}$ & $\begin{array}{l}\text { Para o autor, são fatores de risco: ser solteira, ter baixa escolaridade, baixa renda e falta de acesso ao serviço de } \\
\text { saúde }\end{array}$ \\
\hline
\end{tabular}

Fonte: Autores (2019). 
O Quadro 3 acima traz o nome dos estudos escolhidos e os principais achados encontrados nos trabalhos, os quais são de importância para a realização da discussão.

O Estudo 1 trata-se de uma análise observacional descritiva e exploratória da coorte de mulheres com diagnóstico primário de câncer cervical matriculadas e tratadas num centro que concentra todos os casos de câncer ginecológico do Inca, entre julho de 2012 e outubro de 2014. A população do estudo foi composta pelo universo dos casos de câncer cervical primário - codificado como C53.0 pela 10a Revisão da Classificação Internacional de Doenças (CID10) - com confirmação histopatológica dos seguintes tipos histológicos: escamoso, adenoescamoso e adenocarcinoma. Os critérios de exclusão foram: casos de carcinoma de colo uterino in situ e ter iniciado o tratamento fora do Inca. No período de julho de 2012 a outubro de 2014, foram matriculadas 1.483 mulheres com câncer cervical, no entanto, após a realização dos critérios de exclusão permaneceram no estudo um total de 1.004 pacientes.

No presente estudo, foram analisadas as variáveis sociodemográficas (idade ao diagnóstico de câncer, cor da pele, escolaridade e estado conjugal), reprodutivas (idades da menarca e da sexarca, gestações, abortos, número de filhos e estado menopausal), clínicas (comorbidade, estadiamento tumoral e tipo histológico do tumor) e relacionadas aos hábitos de vida (etilismo, tabagismo e uso de anticoncepcional oral). Aproximadamente $70 \%$ das mulheres foram diagnosticadas com mais de 40 anos de idade em ambos os tipos histológicos, sem diferença estatisticamente significativa nessa distribuição. Houve uma predominância de mulheres não brancas (67,4\%), com menos de 8 anos de estudo (51,9\%), com início da atividade sexual até 16 anos de idade (40,7\%), histórico de gestação (95,5\%), com mais de uma gestação (82,9\%) e mais de dois filhos (52,7\%). Neste estudo, 45,8\% das mulheres com câncer de colo uterino eram tabagistas ou ex-tabagistas.

Conforme o estudo, além da infecção pelo HPV, que é a causa necessária para o câncer de colo uterino, os tipos histológicos Adenocarcinoma Cervical (AC) e Carcinoma de Células Escamosas (SCC) compartilham outros fatores de risco, como o número de parceiros sexuais, idade da primeira atividade sexual, idade da primeira gestação e uso de contraceptivo oral. O tabagismo e a multiparidade são fatores de risco diretamente associados ao SCC e inversamente associados ao AC. Entretanto, alguns autores sustentam que o AC pode representar uma entidade histológica que compartilha dos fatores de risco relacionados ao câncer de endométrio, como a obesidade, o uso de anticoncepcional oral e a nuliparidade, além da associação inversa com o tabagismo. Mulheres nulíparas têm maior número de ciclos menstruais ovulatórios devido à ausência de gravidez e lactação, com maior exposição cumulativa ao hormônio estrogênio e/ou menor exposição ao hormônio progesterona. A progesterona afeta diretamente as células cancerígenas, causando inibição do crescimento das células neoplásicas e invasão celular.

Os resultados encontrados destacam a diferença entre os tipos histológicos, sugerindo que o AC pode representar uma entidade clínica distinta de neoplasia cervical, podendo demandar abordagens distintas das para SCC.

O estudo 2 foi executado em duas fases em que uma ocorreu entre 2010 a 2012, e a segunda fase ocorreu entre 2015 a 2016. A seguinte pesquisa trata-se de um estudo transversal, na qual foi selecionado 778 mulheres submetidas à primeira fase de seguimento entre 20 e 59 anos residentes na área de abrangência de duas Unidades de Atenção Primária à Saúde. Para a segunda fase, foram elegíveis mulheres entre 25 e 64 anos que ainda viviam em território sob a área de abrangência das mesmas UAPS, foram excluídas as histerectomias e as gestantes no período da coleta de dados. As entrevistas foram realizadas por uma equipe de profissionais, em que se utilizaram formulários eletrônicos editados no aplicativo ODK Collect, os questionários abrangeram questões sobre perfil sociodemográfico, autoavaliação do estado de saúde, perfil de acesso aos serviços de saúde, estilo de vida, morbidade, saúde da mulher e comportamento sexual. Ao decorrer do estudo conteve perdas, tanto pelos critérios de exclusão, e também por as mulheres residirem em outro local, a amostra final foi composta por apenas 479 mulheres, das quais $30 \%$ apresentaram alto risco para um exame citopatológico alterado. 
Constatou-se no estudo 2 que as mulheres com baixa renda tiveram a razão de prevalência (RP) em 1,12, com intervalo de confiança (IC) de 95\%: 1,04-1,21, com percepção negativa da própria saúde (RP = 1,13; IC95\%: 1,06-1,20), que faziam uso de tabaco ( $\mathrm{RP}=1,14$; IC95\%: 1,05-1,23) ou álcool ( $\mathrm{RP}=1,09$; IC95\%: 1,02-1,18) apresentaram alto risco de apresentar um exame alterado, ao passo que a idade elevada $(\mathrm{RP}=0,83$; IC95\%: 0,73-0,95) associou-se à diminuição desse risco. Diante do exposto, foi possível constatar que as mulheres jovens, de baixa renda, com percepção negativa da própria saúde e que usavam tabaco ou álcool tinham maior probabilidade de apresentar alterações no exame citopatológico do colo do útero.

O estudo 3 é uma abordagem quantitativa transversal, com o propósito de descrever os modelos que envolvam a coleta de dados em determinantes pontos temporais, assim todos os fenômenos estudados são contemplados durante um período de coleta de dados. O cenário da pesquisa consistiu em uma penitenciaria feminina com capacidade para 300 recolhidas. Por meio do cálculo para populações finitas o tamanho da amostra resultou em 155 mulheres a partir de um tamanho populacional de 258 , intervalo de confiança de $95 \%$, erro máximo de 0,05 e prevalência de 50\%. Não houve perda amostral. A coleta de dados teve início de janeiro a março de 2010. As internas foram convidadas verbalmente a participarem da pesquisa, sendo esclarecidas do objetivo e procedimentos envolvidos. A aceitação foi formalizada com assinatura ou digital de um termo de consentimento livre e esclarecido. Em um formulário estruturado registraram-se informações referentes a caracterização sociodemografica, história sexual e reprodutiva, hábitos tabagistas, idade de início sexual, número de parceiros sexuais, paridade, acompanhamento ginecológico na instituição, uso de preservativo, escolaridade. Riscos relacionado a sexualidade, reprodução e tabagismo: a idade de coitarca demonstrou que 124 iniciaram antes dos 17 anos, sendo que 148 tiveram sua primeira relação sexual na adolescência antes dos 14 anos. O número de parceiros sexuais nos três meses em 81 mulheres relatou ter relação com um único parceiro, de modo que 92 mulheres possuíam de um a três, sendo que 86 mulheres tinham relacionamento estável.

Nas informações obstétricas 65 presidiarias possuíam um a dois filhos e paridade mais elevada de três ou mais foi averiguada em 60 mulheres. Sobre o acompanhamento ginecológico na penitenciaria 65 mulheres confirmam a realização, a maioria não passou por esse serviço após o ingresso na prisão. O hábito de fumar esteve presente em 104 mulheres.

A idade também se configura como interferente no processo do câncer cervicouterino. A maioria das infecções por HPV em mulheres com menos de 30 anos regride espontaneamente, ao passo que acima dessa idade a persistência é mais frequente. A incidência do câncer cervicouterino manifesta-se a partir da faixa etária de 20 a 29 anos, aumentando seu risco rapidamente até atingir o pico etário entre 50 e 60 anos. O risco de mulheres serem acometidas por câncer cervical aumenta entre as que dispõem de baixo nível escolar e socioeconômico. A alta porcentagem de mulheres de pouca instrução pode agravar as vulnerabilidades individuais e coletivas, compondo um fator de risco social para o desenvolvimento do câncer cervicouterino. Somado ao baixo nível educacional e econômico, ser solteira, como observado predominantemente, pode agravar a vulnerabilidade às IST'S/HIV e ao câncer do colo do útero devido a possibilidade de mais variedade de parceiros sexuais.

A precocidade das relações sexuais está diretamente relacionada com o aumento do risco do câncer cervical, pela imaturidade da cérvice na adolescência, intensa metaplasia, zona de transformação do colo localizada na ectocérvice e pelos níveis hormonais desestabilizados. A multiplicidade de parceiros sem uso de medidas preventivas também compões um fator predisponente para o câncer cervicouterino, pois gera aumento de IST'S, inclusive de HPV. No caso das mulheres estudadas e de seus parceiros sexuais, além dos riscos decorrentes do não uso do preservativo nas visitas íntimas, não há como negar os riscos da não adoção nas relações extraconjugais dentro das instituições prisionais, tanto por parte da mulher quanto por parte do homem que também esteja aprisionado, como fora das instituições por aqueles que estão em liberdade. 
A gravidez também se configura como um fator predisponente a infecção por HPV, devido em parte a diminuição da imunidade celular e modificações dos níveis dos hormônios esteroides. Na amostra do estudo 125 passaram pela gravidez e cerca $40 \%$ relataram ter três ou mais filhos. As mulheres, nos primeiros meses de permanência do presidio, realizam o exame preventivo e todas as informações são registradas no prontuário de cada detenta. O tabaco diminui significativamente a quantidade e função das células de Langherans, que são responsáveis pela defesa do tecido epitelial, além do cigarro conter mais de 300 substâncias com potencial efeito cancerígeno.

Como conclusão do estudo 3 foi possível observar que os fatores sociodemográficos das presidiarias revelam uma maioria jovens, solteiras, com baixa escolaridade e renda familiar. Tendo como riscos sexuais e reprodutivos a precocidade do coitarca, o não uso de preservativo, a paridade e a baixa cobertura do rastreamento nas instituições prisionais. A multiplicidade de parceiros nos últimos três meses não se mostrou predisponente, pela estabilidade e pouca variedade de parceiros sexuais da amostra. Hábitos tabagistas estiveram presentes em $70 \%$ das mulheres. A carência ou inexistência de programas multidisciplinares de combate ao tabaco nas comunidades e nas instituições prisionais agrava essa situação. Os fatores avaliados indicam que essas mulheres são vulneráveis a desenvolver a neoplasia estudada.

No estudo 4 mostra que o início da vida sexual de jovens adolescentes é marcado pela grande vulnerabilidade das mesmas e adquirir Infecções sexualmente transmissível ou as conhecidas IST's, uma etapa onde o corpo da mulher sofre mudanças que a adequam para vida adulta. As próprias doenças em pessoas infectadas causam problemas maiores, pois podem gerar lesões, tumores e mesmo em alguns casos, o câncer. Mulheres jovens são muito suscetíveis a adquirir câncer do colo do útero, onde as taxas de sobrevivências ainda não são altas.

Além de fisiologicamente a jovem adolescente nem sempre estar com o corpo apto ao sexo, pois em seu corpo ainda decorrem mudanças, o seu útero pode se tornar mais frágil e adquirir facilmente infecções que podem vez ou outra influenciar em problemas de fertilidade no futuro. A atividade biológica na adolescência está nível máximo por conta da adequação do corpo a vida adulta, só com o acréscimo da idade é que se diminui a expectativa de se contrair uma infecção viral, dessa forma o recomendado para adolescentes é o sexo apenas em idade madura do corpo.

Considerando trabalhos publicados e avaliados para estudo se observa que conforme as descrições o exame de Papanicolau e colposcopia são os exames mais comuns e frequentemente realizados por mulheres jovens durante o período de maior atividade sexual. Se observa também que chega a $82 \%$ a taxa de adolescentes que contraem infecções, problema que causa lesões de baixo grau, sendo que a maioria regride, enquanto uma minoria sofre lesão de alto grau sofrendo lesões mais sérias, essa não é uma tendência só no Brasil, mas sim, uma tendência mundial.

Assim se entende que a adolescente precisa buscar sua própria prevenção e garantir maiores cuidados com seu corpo pois algumas lesões adquirias enquanto jovem pode causar problemas para ela na vida adulta, por isso em caso afirmativo de lesões se deve buscar por ajuda e acompanhamento de profissionais qualificados para acompanhar a evolução da lesão e garantir a sua cura, tendo sempre o cuidado de realizar exames periódicos.

No estudo 5 mostras com relação aos fatores de risco e proteção à saúde de mulheres para prevenção do câncer uterino, em que é válido destacar que se objetiva investigar os riscos da doença e como prevenir a mesma de forma a ajudar na redução do número de casos em Teresina - PI. A maior parcela das mulheres que estão sujeitas a doença está com faixa de idade entre 25 a 39 anos, onde dessas, 78, 5\% buscam por serviços de saúde.

O câncer uterino ou cérvicouterino é um problema de saúde grave que causa alta mortalidade em mulheres de camadas mais pobres da sociedade brasileira, se estima que no ano de 2012 dos 17.540 casos, 100 são diagnosticados apenas na cidade de Teresina, com a aplicação de um tratamento eficaz as chances de cura são altas.

O grande problema está em como trazer mulheres mais pobres para via a realizar exames precoces para descobrir a doença em estágio inicial, normalmente quando é descoberto a doença ela já se encaminha para estágio avançado reduzindo as 
expectativas do tratamento, o ideal é que as mulheres busquem realizar exames do colo de útero e Papanicolau uma vez por ano, após o segundo ano de exames e nada constatado, realizar novamente a cada três anos, para assim melhor avaliar a possibilidade de manifestação da doença.

A principal característica das mulheres que estão dentro da maior incidência de câncer de útero, são, baixa escolaridade, baixa renda, pouco acesso a serviços de saúde, adolescentes, fumam demasiadamente além de consumir em alguns casos entorpecentes. Muitos fatores internos e externos colaboram para que a doença aumente sua incidência, doenças sexualmente transmissíveis, tabagismo estresse emocional e má alimentação são as formas mais comuns onde a doença encontra forças para se alastrar pelo corpo da mulher.

Em Teresina no Piaú, embora a cidade tenha bom aparato médico reconhecido em sua região, ainda é preciso direcionar o ensino nas faculdades locais para uma melhor interação com este tema, pois a teoria de ensino está um pouco diferenciada da realidade e necessidade local. Para melhor exemplificar as noções de tratamento e desenvolvimento da doença na cidade, foi distribuído os resultados obtidos no estudo na Tabela 1 abaixo:

Tabela 1. principais fatores associados ao risco e prevenção do câncer de útero na cidade estudada.

\begin{tabular}{ll}
\hline Local da coleta de dados & Teresina - PI \\
\cline { 2 - 2 } Tipo de doença & Câncer de Útero \\
\cline { 2 - 2 } Principais causas & Tabagismo; \\
& Entorpecentes; \\
& Falta de acesso ao sistema de saúde; \\
Pessoas acometidas & Estresse emocional. \\
\cline { 2 - 2 } Formas de combate & Mulheres entre 25 e 39 anos \\
\cline { 2 - 2 } & Alimentação adequada, consulta exames periódicos e \\
\hline
\end{tabular}

Fonte: Autores (2019).

Assim foram avaliadas para pesquisa, mulheres que tinham entre 25 e 64 anos respectivamente para avaliar padrões de comportamento e utilização publica de serviços de saúde, os dados que serão destacados foram colhidos através de pesquisa locais.

Para uma população de 2000 mulheres o ideal é que ao menos 600 realizem exames periódicos. Além da própria pesquisa e suas características foram avaliados pontos como IMC, remuneração, idade, família, patologias e alcoolismo, se percebendo que das mulheres que participaram da pesquisa do artigo através da assinatura do termo de consentimento tinham como principais características, idade flutuante entre 25 e 39 anos, escolaridade em parte incompleta, tendo entre 9 a 12 anos de estudo continuo, a maioria se declarava casada e as demais solteiras ou separadas. Trabalhavam, se deslocavam com uso de transporte público, foro isto o segundo meio utilizado era moto convencional ou bicicleta.

A mulheres possuíam renda per capita entre 678 reais e 1 salário e meio, tendo acesso limitado a serviços de saúde, a maior parte busca consultas por ginecologistas em agencias de saúde nos bairros, boa parte de suas consultas fora busca por ginecologista são ligadas a problemas diabéticos, hipertensão e problemas psiquiátricos onde pouco mais da metade delas recebe orientação coerente sobre a coleta para exame.

Conclui- se que de acordo com todos os resultados logrados nesse estudo de caso, o controle da manifestação do câncer de útero denota de melhorias em campanhas educacionais e maior incentivo a participação da mulher na manutenção de sua saúde. 


\section{Conclusão}

Os objetivos de estudo foram alcançados de forma satisfatória, pois foi possível descrever que o uso de cigarro, multiplicidade de parceiros sexuais, multiparidade, baixa escolaridade, início precoce das atividades sexuais, presença de IST'S, uso de anticoncepcionais orais e a obesidade são fatores de risco para câncer do colo do útero. Os resultados mostram que esses fatores têm alta evidência para o desenvolvimento do câncer.

Um dos desafios encontrados foi a escassez de artigos relacionado ao tema, no entanto faz-se necessário que haja mais estudo a respeito dessa proposta, para ter uma melhor dimensão do mesmo. Espera-se que esse trabalho possa contribuir para o campo científico com o intuito de detectar os fatores de risco e poder intervir prevenido o câncer do colo do útero. Como sugestões futuras, a pesquisa visa abrir caminhos para que sejam realizadas mais buscas em relação a temática proposta pelo artigo.

\section{Referências}

Brazil, S. (2017). Sobrevida de mulheres com câncer de colo uterino em um centro de referência do sul do Brasil. Braz J Oncol, $13(46)$, 1-7.

de Oliveira, A. C., Pessoa, R. S., de Carvalho, A. M. C., \& Magalhães, R. D. L. B. (2014). Fatores de risco e proteção à saúde de mulheres para prevenção do câncer uterino. Rev Rene, 15(2), 240-248.

do Carmo Rocha, J., Bustamante Teixeira, M. T., Azevedo e Silva, G., de Castro Dias, K., \& Salim Miranda Duque, M. L. (2014). Prevalence of prehypertension and associated factors in women. Investigacion y educacion en enfermeria, 32(3), 471-479.

Ercole, F. F., Melo, L. S. D., \& Alcoforado, C. L. G. C. (2014). Revisão integrativa versus revisão sistemática. Revista Mineira de Enfermagem, 18(1), 9-12.

Lombelo-Campos, A. A., Neves, F. S., Duque, K. D. C. D., Leite, I. C. G., Guerra, M. R., \& Teixeira, M. T. B. (2018). Fatores associados ao risco de alterações no exame citopatológico do colo do útero. Revista de Enfermagem do Centro-Oeste Mineiro, 8.

Macêdo, F. L. D. S., Silva, E. R. D., Soares, L. R. C., Rosal, V. M. D. S., Carvalho, N. A. L., \& Rocha, M. G. D. L. (2015). Infecção pelo HPV na adolescente. Femina, 185-188.

Ministério da Saúde (MS). (2015). Secretaria de Vigilância em Saúde. Secretaria de Atenção à Saúde. Política Nacional de Promoção da Saúde (PNPS): revisão da Portaria MS/GM $n^{\circ}$ 687, de 30 de março de 2006.

Nicolau, A. I. O., Aquino, P. D. S., Ximenes, L. B., \& Pinheiro, K. B. (2015). Determinantes sociais proximais relacionados ao câncer cervicouterino em mulheres privadas de liberdade. REME rev. min. enferm, 733-740.

Rozario, S. D., Silva, I. F. D., Koifman, R. J., \& Silva, I. F. D. (2019). Caracterização de mulheres com câncer cervical atendidas no Inca por tipo histológico. Revista de Saúde Pública, 53, 88.

Soares, C. B., Hoga, L. A. K., Peduzzi, M., Sangaleti, C., Yonekura, T., \& Silva, D. R. A. D. (2014). Revisão integrativa: conceitos e métodos utilizados na enfermagem. Revista da Escola de Enfermagem da USP, 48(2), 335-345.

Vukovic, D., Antic, L., Vasiljevic, M., Antic, D., \& Matejic, B. (2015). Development of a risk index for prediction of abnormal pap test results in Serbia. Asian Pacific Journal of Cancer Prevention, 16(8), 3527-3531. 